Third Meeting, 10th January 1902.

Dr ThIRd, Vice-President, in the Chair.

\title{
Note on the Use of Fourier's Series in the Problem of
} the Transverse Vibrations of Strings.

\section{By H. S. CarsLaw.}

$\$ 1$.

Fourier's Series were first applied to the solution of the Differential Equations which occur in the Theory of the Conduction of Heat and that of the Vibrations of Stretched Strings, namely,

$$
\begin{aligned}
\frac{\partial v}{\partial t} & =\kappa \frac{\partial^{2} v}{\partial x^{2}}, \\
\frac{\partial^{2} y}{\partial t^{2}} & =a^{2} \frac{\partial^{2} y}{\partial x^{2}} .
\end{aligned}
$$

In the first, when the flow of heat is linear and the initial temperature is given in the range $o<x<\pi$ by $f(x)$, the solution can be put in the form

$$
v=\frac{2}{\pi} \sum_{1}^{\infty} e^{-\kappa n^{2} t} \sin n x \int_{0}^{\pi} f\left(x^{\prime}\right) \sin n x^{\prime} d x^{\prime} \quad-\quad-
$$

In this case there is no difficulty with regard to the differentiation of the infinite series, term by term, as the factor $e^{-\kappa n^{2} t}$ causes the series which we thus obtain to remain uniformly convergent with respect to $x$ and $t$ in the intervals concerned.

The corresponding problem in the Theory of the Transverse Vibrations of Strings we shall simplify by taking the initial velocity of the string as zero, the string being stretched between $x=0$ and $x=\pi$, and displaced at $t=0$ into the form $y=f(x)$.

The solution of this problem is

$$
y=\frac{2}{\pi} \Sigma \sin n x \cos n a t \int_{0}^{\pi} f\left(x^{\prime}\right) \sin n x^{\prime} d x^{\prime}
$$

but the reasoning by which this is usually established is not valid. 
The convergence factor which we noticed in the case above does not now occur, and, as a matter of fact, the series in (2), when the initial position of the string has not a continuous slope, is not differentiable term by term. This was pointed out for the case given in Rayleigh's Sound, Vol. II., p. 185, where the string is plucked aside at the point $x=b$ through a distance $\gamma$, by Lindemann * the same problem is discussed at length by Harnack $\dagger$ and Morera + ; but the points raised seem to have escaped the attention of English writers on this subject. In the special case of Rayleigh's problem the solution is given by

$$
y=\frac{2 \gamma}{b(\pi-b)} \sum_{1}^{\infty} \frac{1}{n^{2}} \sin n b \sin n x \cos n a t
$$

and it is obvious that the series we obtain by differentiating the right hand of (3) term by term twice with regard to $x$ or $t$ are not convergent. Indeed the terms as $n$ increases do not ultimately vanish. Hence the new series do not represent $\frac{\partial^{2} y}{\partial t^{2}}$ and $\frac{\partial^{2} y}{\partial x^{2}}$, which in this case are both zero, as the string remains made up of straight lines. It is not a proof to say that the function given by (3) satisfies our equation, since each of its terms $\sin n x \cos n a t$ does so. If the series we obtained by differentiating (3) term by term were uniformly convergent in the intervals concerned, this reasoning would hold, but not otherwise.

The object of this paper is to show that the trigonometrical solution of any problem, where the initial position of the string forms a curve in which the slope is at one or more points discontinuous and sharp corners occur, involves an infinite series in which

* Lindemann,

Die Schwingungsformen gezupfter und gestrichener Saiten.

Berichte über die Verhandlungen der naturforschender Gesellschaft zur Freiburg. B. VII. 1879.

This paper is translated in the Philosophical Magazine for 1880, under the title "On the Forms of the Vibrations of Twitched and Stroked Strings."

t Harnack, Über die mit Ecken behafteten Schwingungen gespannter Saiten. Math. Annalen, XXIX.

$\ddagger$ Morera,

Sul Problema delle Corde Vibrante.

Atti della Academia delle Scienze di Torino. T. XXIII. 
the terms are of the order $\frac{1}{n^{2}}$, and which therefore can not be differentiated term by term twice with regard to $x$ or $t$; that when there are no such corners the terms are of order $\frac{1}{n^{3}}$, and the term by term differentiation is possible; and to point out that the simplest demonstration is the reduction of the Trigonometrical Solution to the form given by D'Alembert, in which the functional nature of the expression for $y$ at once shows that it is a solution of the equation.

Returning to the equation

$$
\$ 2 .
$$

$$
y=\frac{2}{\pi} \Sigma \sin n x \cos n a t \int_{0}^{\pi} f\left(x^{\prime}\right) \sin n x^{\prime} d x
$$

we may break up the right-hand side into the sum of the two convergent series

$$
\begin{aligned}
& \frac{1}{\pi} \Sigma \sin n(x+a t) \int_{0}^{\pi} f\left(x^{\prime}\right) \sin n x^{\prime} d x^{\prime} \\
& \text { and } \frac{1}{\pi} \Sigma \sin n(x-a t) \int_{0}^{\pi} f\left(x^{\prime}\right) \sin n x^{\prime} d x^{\prime},
\end{aligned}
$$

which represent $\frac{1}{2} f(x+a t)$ and $\frac{1}{2} f(x-a t)$, respectively.

$$
\text { Hence } \quad y=\frac{1}{2}[f(x+a t)+f(x-a t)]
$$

and this expression obviously satisfies the equation

$$
\frac{\partial^{2} y}{\partial t^{2}}=a^{2} \frac{\partial^{2} y}{\partial x^{2}} \text {. }
$$

As $f(x)$ is necessarily a continuous function, the series (4) and (5) are also continuous, and the conditions at $x=0, x=\pi$, and $t=0$ are therefore fulfilled. Our solution is thus verified without entering upon the question of the possibility of the differentiation of the Infinite Series,

$$
\$ 3 .
$$

We shall now show that when $f^{\prime}(x)$ is discontinuous at one or more points between $x=0$ and $x=\pi$, the terms of the series (2) are of the order $\frac{1}{n^{2}}$, and that when it is continuous the terms are of order $\frac{1}{n^{3}}$. 
Taking the representative term, namely,

$$
\int_{0}^{\pi} f\left(x^{\prime}\right) \sin n x^{\prime} d x^{\prime}
$$

and integrating by parts, we obtain

$$
-\frac{1}{n} \cos n x^{\prime} f\left(x^{\prime}\right)+\frac{1}{n} \int f^{\prime}\left(x^{\prime}\right) \cos n x^{\prime} d x^{\prime},
$$

and when we insert the limits, and note that $f(x)$ vanishes at $x=0$ and $x=\pi$, we see that this reduces to

$$
\frac{1}{n} \int_{0}^{\pi} f^{\prime}\left(x^{\prime}\right) \cos n x^{\prime} d x^{\prime}
$$

Integrating again by parts, we obtain

$$
\frac{1}{n^{3}} \sin n x^{\prime} f^{\prime}\left(x^{\prime}\right)-\frac{1}{n^{2}} \int f^{\prime \prime}\left(x^{\prime}\right) \sin n x^{\prime} d x^{\prime} ;
$$

and if $f^{\prime}(x)$ is discontinuous at points between 0 and $\pi$, our integral has to be broken up into the corresponding intervals, and the first part of this expression does not vanish. Therefore, when sharp corners occur in the initial position, the $n^{\text {th }}$ term of our series is of the order $\frac{1}{n^{2}}$.

If $f^{\prime}(x)$ had been continuous, the term would have reduced to

$$
-\frac{1}{n^{2}} \int_{0}^{\pi} f^{\prime \prime}\left(x^{\prime}\right) \sin n x^{\prime} d x^{\prime}
$$

When we apply the Second Theorem of Mean Value to this integral, we see that

$$
\begin{gathered}
\int_{0}^{\pi} f^{\prime \prime}\left(x^{\prime}\right) \sin n x^{\prime} d x^{\prime}=f^{\prime \prime}(0) \int_{0}^{\xi} \sin n x^{\prime} d x^{\prime}+f^{\prime \prime}(\pi) \int_{\xi}^{\pi} \sin n x^{\prime} d x^{\prime} \\
=\frac{1}{n}\left\{f^{\prime \prime}(0)(1-\cos n \xi)+f^{\prime \prime}(\pi)(\cos n \xi-\cos n \pi)\right\}
\end{gathered}
$$

where $\xi$ is some value of $x$ between 0 and $\pi$; and in this case, the terms are of the order $\frac{1}{n^{3}}$.

\section{$\$ 4$.}

When the $n^{\text {th }}$ term is of the order $\frac{1}{n^{2}}$, the second differential coefficients with respect to $n$ and $t$ will be of the order $\frac{1}{n}$; and it is not at once clear whether the series we obtain by term to term 
differentiation will represent the differential coefficients of the given function; that they will generally do so in this case can be shown in the following way.

When $f^{\prime \prime}(x)$ satisfies the conditions which admit of expansion in Fourier's Series, the series

$$
\Sigma \sin n x \int_{0}^{\pi} f^{\prime \prime}\left(x^{\prime}\right) \sin n x^{\prime} d x^{\prime}
$$

is uniformly convergent, in general, that is, except in intervals which include points of discontinuity of $f^{\prime \prime}(x)$.

But

$$
\begin{gathered}
\int f^{\prime \prime}\left(x^{\prime}\right) \sin n x^{\prime} d x^{\prime} \\
=\left[f^{\prime}\left(x^{\prime}\right) \sin n x^{\prime}\right]-n \int f^{\prime}(x) \cos n x^{\prime} d x^{\prime}
\end{gathered}
$$

where the limits in the case when $f^{\prime}(x)$ is discontinuous would have to be those of the intervals into which we would break up the range of integration. However, if $f^{\prime}(x)$ is continuous, and this is the case with which we are dealing, our result simplifies, and we see that

$$
\begin{aligned}
& \int_{0}^{\pi} f^{\prime \prime}\left(x^{\prime}\right) \sin n x^{\prime} d x^{\prime} \\
= & -n \int_{0}^{\pi} f^{\prime}\left(x^{\prime}\right) \cos n x^{\prime} d x^{\prime} \\
= & -n\left[f\left(x^{\prime}\right) \cos n x^{\prime}\right]_{0}^{\pi}-n^{2} \int_{0}^{\pi} f\left(x^{\prime}\right) \sin n x^{\prime} d x^{\prime} \\
= & -n^{2} \int_{0}^{\pi} f\left(x^{\prime}\right) \sin n x^{\prime} d x^{\prime} .
\end{aligned}
$$

Hence the terms of the series we obtain by term to term differentiation of (2) are equal in magnitude and opposite in sign to those of the uniformly convergent series for $f^{\prime \prime}(x)$, and these series represent the differential coefficients of the function.

$\$ \mathbf{b}$.

The questions discussed in the preceding paragraphs are of pure mathematical interest. There is a dynamical difficulty in the case in which sharp corners occur in the initial, and therefore, according to our solution, in the changing form of the string : for the equation

$$
\frac{\partial^{2} y}{\partial t^{2}}=a^{2} \frac{\partial^{2} y}{\partial x^{2}}
$$

is obtained on the assumption that no such discontinuities occur. 
Although this point is not discussed in any of the English books on the subject, the question was noted by the earliest writers on the subject, among others by D'Alembert,* and was fully solved by Christoffel $\dagger$ in 1876 .

A full treatment is given in the recently published book by Weber, Die Partiellen Differential-Gleichungen der Mathematischen Physik, B. II., \$88.

* See the discussion in Burkhardt's Hntwickelungen nach oscillirenden Functionen, pp. 10-24.

+ Untersuchungen über die mit dem Fortbestehen linearer partieller Differen. tial Gleichungen verträglichen Unstetigkeiten.

Annali di Matematica. T. VIII. 